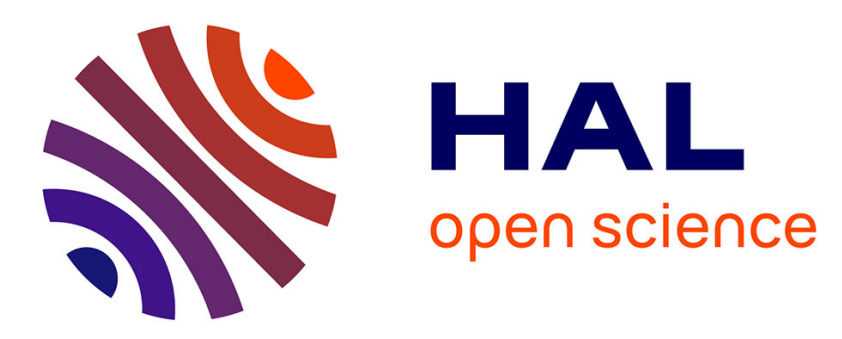

\title{
Two-dimensional numerical analysis of the Poiseuille-Bénard flow in a rectangular channel heated from below
}

\author{
Xavier Nicolas, Abdelkader Mojtabi, J. K. Platten
}

\section{- To cite this version:}

Xavier Nicolas, Abdelkader Mojtabi, J. K. Platten. Two-dimensional numerical analysis of the Poiseuille-Bénard flow in a rectangular channel heated from below. Physics of Fluids, 1997, 9 (2), pp.337-348. hal-00695300

\section{HAL Id: hal-00695300 https://hal.science/hal-00695300}

Submitted on 25 Sep 2014

HAL is a multi-disciplinary open access archive for the deposit and dissemination of scientific research documents, whether they are published or not. The documents may come from teaching and research institutions in France or abroad, or from public or private research centers.
L'archive ouverte pluridisciplinaire HAL, est destinée au dépôt et à la diffusion de documents scientifiques de niveau recherche, publiés ou non, émanant des établissements d'enseignement et de recherche français ou étrangers, des laboratoires publics ou privés. 


\title{
Two-dimensional numerical analysis of the Poiseuille-Bénard flow in a rectangular channel heated from below
}

\author{
X. Nicolas and A. Mojtabi \\ Institut de Mécanique des Fluides, UMR CNRS/INP-UPS 5502, Université Paul Sabatier, UFR MIG, \\ 118 route de Narbonne, 31062 Toulouse Cedex, France \\ J. K. Platten \\ Laboratoire de Chimie Générale, Faculté de Médecine, Université de Mons-Hainaut, 7000 Mons, Belgium
}

(Received 24 June 1996; accepted 22 October 1996)

\begin{abstract}
The Poiseuille-Bénard flow (PBF) is studied by a two-dimensional numerical simulation for a Prandtl number equal to 6.4 (that of water at $23{ }^{\circ} \mathrm{C}$ ) and for a wide range of Rayleigh (Ra) and Reynolds (Re) numbers: $\mathrm{Ra} \leqslant 6000$ and $\mathrm{Re} \leqslant 3$. The two observed flow configurations are (1) thermally stratified Poiseuille flow and (2) thermoconvective transversal rolls superimposed to the basic Poiseuille flow. The time evolution of the velocity components, the spatial development of the transversal rolls, their frequency, wavelength and velocity, the Nusselt number, together with the stability map in the Ra-Re plane, are studied in detail. Whenever possible, quantitative comparisons are made with published results: most of the experimental data, based on laser-Doppler anemometry (LDA), are recovered with amazing accuracy; a good agreement with results of convective stability deduced from a weakly nonlinear Ginzburg-Landau theory is also obtained. (C) 1997 American Institute of Physics. [S1070-6631(97)02102-8]
\end{abstract}

\section{INTRODUCTION}

The Poiseuille-Bénard flow (PBF) is a mixed convection flow in a horizontal rectangular channel heated from below. This problem has been widely studied, particularly because of its practical or technological interest. During this first half century, research on this subject attempted to explain certain meteorological phenomena like the cloudy band alignment under the action of the wind. ${ }^{1,2}$ More recently, applications have been concerned with technological processes like the cooling of electronic components ${ }^{3,4}$ or the production of thin films in CVD (chemical vapor deposition) reactors $;{ }^{5-10}$ these works have mainly focused on the heat transfer enhancement related to thermoconvective structures in the flow. Because of the richness of its dynamical behavior, the PBF has also given rise to fundamental studies on the stability of the different thermoconvective patterns that are liable to arise. The present paper is in keeping with these studies.

The PBF is the result of the superimposition of two convective sources: (1) a horizontal pressure gradient giving rise to a forced flow, characterized by its Reynolds number Re, and (2) a vertical temperature gradient (characterized by its Rayleigh number Ra) the source of thermoconvective structures.

Results of linear hydrodynamic stability theory ${ }^{11-13}$ have shown that the thermally stratified Poiseuille flow (the "basic flow") keeps stable as long as Ra does not exceed a certain critical value Ra* (cf. Fig. 1). Beyond this value, the basic flow becomes unstable and two kinds of thermoconvective structures, called "transversal rolls" and "longitudinal rolls," may appear. The transversal rolls, $R_{\perp}$ (respectively longitudinal rolls, $R_{\|}$) have their axes perpendicular (respectively parallel) to the direction of the mean flow. While the longitudinal rolls are stationary structures, the transversal rolls are carried away out of the channel by the average flow; they can be considered like a quasi-twodimensional (2-D) structure, while in the longitudinal rolls the three velocity components are excited. In the case of ducts of infinite lateral extension (the transversal aspect ratio $B=l / h=\infty$, where $l$ and $h$ are, respectively, the channel width and height), the longitudinal rolls are shown to appear first [Fig. 1(a)], since the critical Rayleigh number for the longitudinal rolls, $\mathrm{Ra}_{\|}^{*}=1708$, is always smaller than $\mathrm{Ra}_{\perp}^{*}$ (the critical Rayleigh number for the transversal rolls). For finite rectangular ducts [Fig. 1(b)], the lateral confinement has two effects: first, it tends to stabilize the basic flow $\left[\right.$ when $B$ decreases, $\mathrm{Ra}^{*}=\min \left(\mathrm{Ra}_{\perp}^{*}, \mathrm{Ra}_{\|}^{*}\right)$ grows; $\left.\mathrm{Ra}^{*}>1708\right]$; second, the vertical lateral boundaries promote the appearance of the transversal rolls at Re smaller than a critical value $\mathrm{Re}^{*}$; when $\mathrm{Re}>\mathrm{Re}^{*}$, the main flow favors the longitudinal rolls. Note that $\mathrm{Ra}_{\perp}^{*}$ depends not only on $\mathrm{Re}$ and $B$, but also on the Prandtl number Pr: when Pr increases, $\mathrm{Ra}_{\perp}^{*}$ increases. Since $\mathrm{Ra}_{\|}^{*}$ is not modified by $\mathrm{Pr}, \mathrm{Re}^{*}$ diminishes when $\operatorname{Pr}$ increases. Thus, for $\operatorname{Pr} \approx 0.7$ (air) $\mathrm{Re}^{*} \approx 7$ (see Refs. 14 and 15 ) and for $\operatorname{Pr} \approx 6.4$ (water) $\mathrm{Re}^{*} \approx 0.3$ (see Refs. 14 and 16). The very small value of Re* explains why, for a long time, only a few works have been devoted to the transversal roll behavior or to the transition $R_{\perp}-R_{\|}$, comparing with the literature dealing with the longitudinal rolls.

It is important to note that the stability diagram in Fig. 1 is the result of a linear analysis, only valid near the critical Rayleigh number $\mathrm{Ra}^{*}$. When investigating the nonlinear behavior of the PBF, the structure of the flow becomes much more complex. Experimental works by Ouazzani et al. ${ }^{14,16,17}$ or by Chiu and Rosenberger, ${ }^{9}$ recent 3-D numerical simulations $^{15,18}$ and studies based on a weakly nonlinear Ginzburg-Landau model ${ }^{19,20}$ have shown that the transition $R_{\perp}-R_{\|}$is not as sharp as it is represented in Fig. 1. Near the triple transition point K [Fig. 1(b)], the transversal and longitudinal rolls compete and periodic or intermittent patterns 

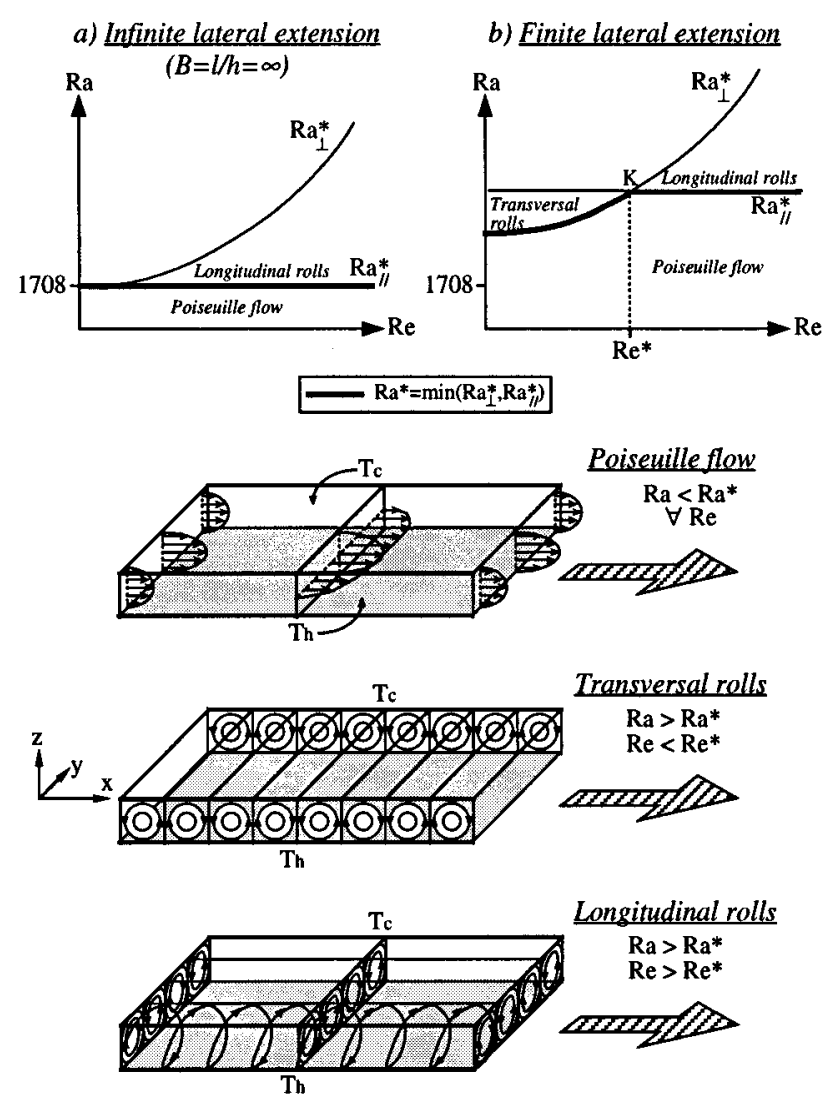

FIG. 1. Schematic presentation and stability diagram (result of the linear stability theory) of different configurations encountered in the PBF; (a) PBF between two infinite horizontal plates; (b) PBF in a channel with infinite longitudinal aspect ratio and finite lateral aspect ratio.

can arise. ${ }^{17}$ Furthermore, in some conditions, transversal or longitudinal rolls, can be observed for the same set of the dimensionless parameters, according to the initial conditions.

Considering first only transversal rolls, Müller et al. ${ }^{21}$ have applied the concept of convective instability in the PBF and defined a new critical Rayleigh number for the transversal rolls, $\mathrm{Ra}_{\perp}^{\text {conv }}>\mathrm{Ra}_{\perp}^{*}$ (Fig. 2). When $\mathrm{Ra}_{\perp}^{*}<\mathrm{Ra}<\mathrm{Ra}_{\perp}^{\text {conv }}$, the flow is convectively unstable: a local perturbation, appearing at time $t_{0}$ at $x=x_{0}$ (cf. Fig. 2), will be allowed to increase with time in a moving frame of reference, but it will be

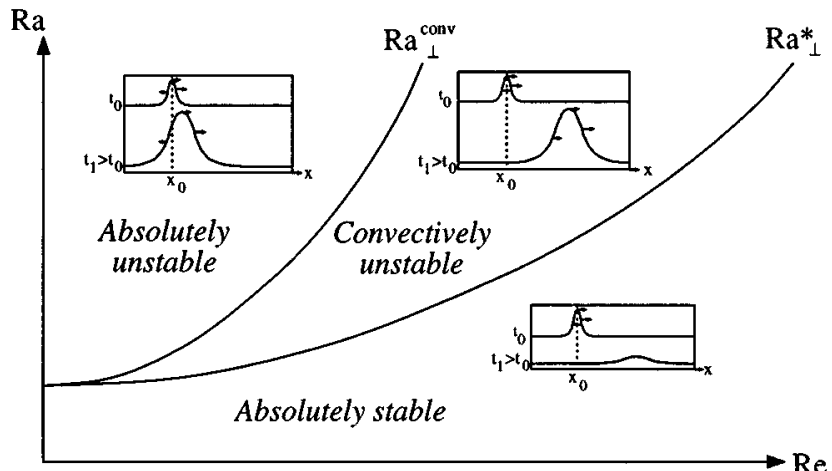

FIG. 2. Critical Rayleigh numbers for the transversal roll configuration according to the linear and convective stability criteria; schematic presentation of the space and time evolution of a small perturbation in the cases of absolutely stable flows and convectively and absolutely unstable flows. damped, at each point of the duct, for a long enough time. ${ }^{22}$ In our case and in the domain of convective instabilities, it will be necessary to sustain a perturbation by a forcing (or a white noise) to create a global pattern, i.e., transversal rolls. When $\mathrm{Ra}>\mathrm{Ra}_{\perp}^{\text {conv }}$, the flow is absolutely unstable: any local perturbation (Fig. 2) will grow at all points of the duct until it asymptotically reaches saturation and the establishment of the transversal rolls.

In their study, Müller et al. ${ }^{21}$ carry out a 2-D numerical simulation of the PBF, for the transversal roll configuration and for $\operatorname{Pr}=1$, in order to validate the Ginzburg-Landau amplitude equation. Recently, Ouazzani et $_{\text {al. }}{ }^{23}$ have adapted the results of the preceding study to the case of water ( $\mathrm{Pr}$ $=6.4$ ) to compare with experiments based on LDA investigations; they show that the transition between the thermally stratified Poiseuille flow and the transversal rolls favorably compares with $\mathrm{Ra}_{\perp}^{\text {conv }}$, but not with $\mathrm{Ra}_{\perp}^{*}$ : the transition closely corresponds to the convective instability curve, not to the neutral one.

In the present paper results are reported on the transversal roll behavior obtained by a 2-D direct numerical simulation. A part of these results refer to the experiments of Ouazzani et al. ${ }^{14,16}$ in order to quantitatively compare experiments and theory. Therefore, the Prandtl number of the fluid is equal to 6.4; the flow is systematically studied for Reynolds and Rayleigh numbers such that $\operatorname{Re}<3$ and $\operatorname{Ra}<6000$. Thus, all the presented results cover a wide range of dimensionless parameters, from the linear to the nonlinear domain. Whenever possible, comparisons with the studies of Müller et al. ${ }^{21,23,24}$ are also given. The specific problems linked with the numerical simulation of convective patterns and open flows on finite computational domains are presented; in particular, the influence of the boundary conditions at the outlet of the channel and that of the periodic boundary conditions will be dealt with.

After having presented the methodology used to compute the PBF, the results are discussed in five distinct parts.

First, quantitative comparisons with Ouazzani's experiments, are made concerning the evolution of the vertical velocity component $W$ with Ra and Re; most of the experimental measurements of this velocity component, obtained by LDA in Ref. 16, are numerically reproduced with amazing accuracy. Simultaneously, the horizontal velocity component, $U$, and the average Nusselt number, $\mathrm{Nu}$, on the top and bottom plates for the fully established transversal roll flow, are recorded and discussed.

Then, for numerous values of $\mathrm{Ra}$ and $\mathrm{Re}$, the space development of the transversal rolls is visualized by means of a stationary intensity envelope of $W$. The characteristic growth length, $l_{e}$, of the transversal rolls is deduced from these envelopes and is shown to be in very good agreement with the result obtained by the Ginzburg-Landau theory. ${ }^{23}$

In the next part, the stability map of the 2-D numerical PBF is presented. It shows the transition between the Poiseuille flow and the transversal rolls (the only two configurations that can be observed by 2-D simulation) in the $\mathrm{Ra}-\mathrm{Re}$ plane. $\mathrm{Ra}_{\perp}^{*}, \mathrm{Ra}_{\perp}^{\mathrm{conv}}$ and the different convective patterns encountered in Ouazzani's experiments are projected 
on this map. The good agreement with the criterion of convective instability can be verified.

Then, the space and time average Nusselt number, $\langle\mathrm{Nu}\rangle$, is favorably compared with a theoretical formula given by Müller ${ }^{24}$ and valid on a weakly nonlinear domain (Ra $<2500$ ). The numerical values of $\langle\mathrm{Nu}\rangle$ are obtained from two different configurations of the computational domain, using two different kinds of inlet and outlet boundary conditions. By means of the Nusselt number, the transition from the transversal rolls to the Poiseuille flow is shown to satisfy the criterion of convective stability when open boundary conditions (OBC) are used at the outlet of the domain, whereas the criterion of linear stability is verified when periodic boundary conditions are imposed.

Finally, we focus on the transversal roll frequency $f$, wavelength $\lambda$, and velocity. To our knowledge, the present work is one of the few studies dealing with the wavelength evolution in the PBF, for a wide range of the parameters in a nonlinear domain. On the other hand, it is well known $n^{4,12,14-16,18,25}$ that the transversal roll velocity, $V r$, can be from $10 \%$ to $50 \%$ higher than the average velocity, $U^{\circ}$, of the flow; the ratio $\mathrm{Vr} / U^{\circ}$ is also shown to decrease linearly with Ra, but to be independent of Re. In this paper, we present several results for $\operatorname{Vr} / U^{\circ}$ at $\operatorname{Pr}=6.4$ and we show that it is possible to precisely reproduce the results obtained by the Ginzburg-Landau model. ${ }^{24}$

\section{METHODOLOGY}

The numerical code used to simulate the 2-D PBF solves the three conservation equations (mass, momentum, and energy) on a rectangular domain of length $L$ and height $h$, uniformly heated from below (at temperature $T_{h}$ ) and cooled from above (at temperature $T_{c}$ ); the no slip boundary conditions are applied to the velocity on the top and bottom plates. A Newtonian and incompressible fluid is considered and the Boussinesq approximation is assumed to be valid. Thus, the dimensionless governing equations in primitive variables (velocity vector $\mathbf{V}$, pressure $P$, temperature $T$ ) can be written as

$$
\begin{aligned}
& \boldsymbol{\nabla} \cdot \mathbf{V}=0, \\
& \frac{\partial \mathbf{V}}{\partial t}+(\mathbf{V} \cdot \boldsymbol{\nabla}) \mathbf{V}=-\boldsymbol{\nabla} P+\frac{1}{\operatorname{Re}} \Delta V+\frac{\mathrm{Ra}}{\operatorname{Re}^{2} \operatorname{Pr}} T \mathbf{k}, \\
& \frac{\partial T}{\partial t}+\mathbf{V} \cdot \boldsymbol{\nabla} T=\frac{1}{\operatorname{Re} \operatorname{Pr}} \Delta T,
\end{aligned}
$$

where the characteristic length, velocity, pressure, and temperature for scaling are $h, U^{\circ}, \rho\left(U^{\circ}\right)^{2}$, and $\left(T_{h}-T_{c}\right)$, respectively. Therefore, the Reynolds number $\operatorname{Re}=U^{\circ} h / \nu$, the Rayleigh number $\mathrm{Ra}=g \beta\left(T_{h}-T_{c}\right) h^{3} / \nu \alpha$, and the Prandtl number $\operatorname{Pr}=\nu / \alpha$. Here $\rho$ is the mass per unit volume, $\nu$ the kinematic viscosity, $g$ the gravitational acceleration, $\beta$ the thermal expansion coefficient, and $\alpha$ the thermal diffusivity. ${ }^{26}$ In addition, $\mathbf{k}$ is the upward vertical unit vector.

The numerical code used to treat the incompressibility constraint and the velocity-pressure coupling between the mass and momentum equations, is based on the augmented lagrangian method ${ }^{27,28}$ and the use of a Uzawa-type

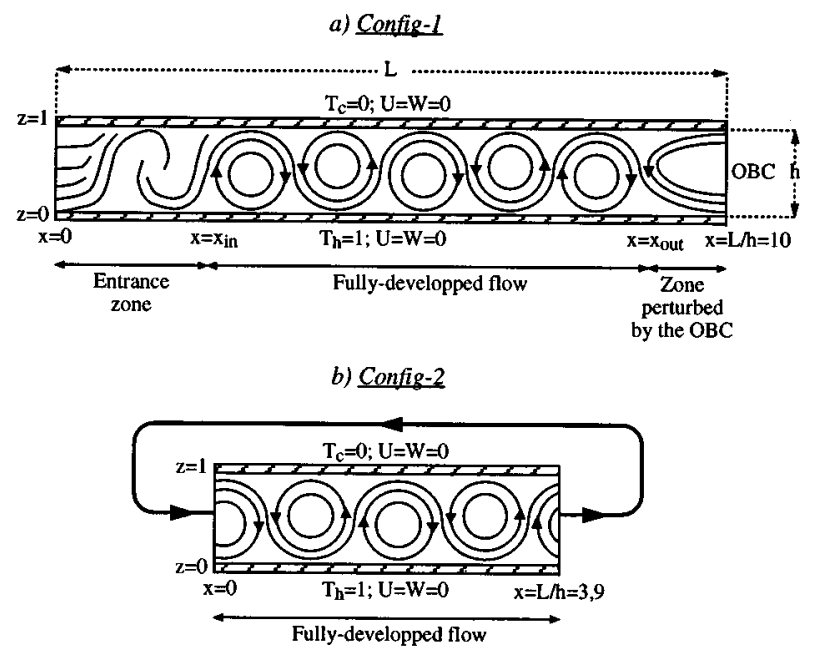

FIG. 3. Schematic presentation of the transversal roll development and of the boundary conditions for the two main computational configurations used; (a) config-1: thermally stratified Poiseuille flow at the inlet and open boundary condition at the outlet; (b) config-2: periodic boundary conditions.

algorithm. ${ }^{29}$ (For more details about the method and the numerical aspects see Ref. 30.) The equations are discretized by a finite volume method on a staggered grid. The convective terms are discretized by a second-order centered differencing and the diffusive terms are approximated by secondorder centered derivatives. The time scheme is Gear's second-order backward implicit scheme; the time step $\Delta t$ $=0.0005$ is used for all the unsteady computations. The linear systems are solved with a preconditioned conjugate gradient method. ${ }^{30}$

All the computations have been realized on the same geometrical configuration (noted config-1) but, in a few cases, two other configurations (config-2 and config-3) have been studied.

Config-1: [Fig. 3(a)] this is a ten aspect ratio duct $(A$ $=L / h=10$ ). (However, in some cases clearly mentioned, this aspect ratio will be equal to 20.) The inlet boundary conditions are a Poiseuille profile for velocity and a linear profile for temperature. At the outlet boundary, several OBC have been tested and compared; ${ }^{30}$ an Orlanski ${ }^{31}$-type boundary condition has been chosen allowing the smallest amplitude perturbation at the outlet. The boundary conditions are summarized in Table I.

All the computations with config-1 have been achieved with the following space steps in the axial and spanwise directions: $(\Delta x ; \Delta z)=(0.1 ; 0.05)$. This configuration allows

TABLE I. Boundary conditions used to compute the PBF with config-1.

\begin{tabular}{llll}
\hline \hline Inlet $(x=0)$ & Bottom $(z=0)$ & Top $(z=1)$ & Outlet $(x=L / h)$ \\
\hline$U(0, z, t)=6\left(z-z^{2}\right)$ & $U(x, 0, t)=0$ & $U(x, 1, t)=0$ & $\frac{\partial U}{\partial t}+U^{0} \frac{\partial U}{\partial x}=0$ \\
$W(0, z, t)=0$ & $W(x, 0, t)=0$ & $W(x, 1, t)=0$ & $\frac{\partial W}{\partial t}+U^{0} \frac{\partial W}{\partial x}=0$ \\
$T(0, z, t)=1-z$ & $T(x, 0, t)=1$ & $T(z, 1, t)=0$ & $\frac{\partial T}{\partial t}+U^{0} \frac{\partial T}{\partial x}=0$ \\
\hline \hline
\end{tabular}


us to observe the space amplification of the perturbation until nonlinear saturation occurs; when thermoconvection develops in the PBF, three zones can be distinguished [Fig. 3(a)]: (1) for $0 \leqslant x \leqslant x_{\text {in }}$, the entrance zone in which the perturbation is growing; then, after its saturation, (2) for $x_{\text {in }} \leqslant x \leqslant x_{\text {out }}$, a fully established periodic flow of transversal rolls; and (3) near the outlet, for $x_{\text {out }} \leqslant x \leqslant L / h$, a small zone where the rolls are slightly distorted by the OBC. In most of our simulations, the length of this third zone is smaller than $h$ but can be higher in a few runs due to its divergence at the critical point. ${ }^{30}$ Note that, for a fixed Rayleigh number, the length of the entrance zone increases when the Reynolds number increases; sometimes, transversal rolls do not even appear in the domain of computation at high Reynolds numbers, especially for small Rayleigh numbers. Numerically, this configuration allows us to compute the characteristic growth length, $l_{e}$, of the transversal rolls, and consequently, to determine $\mathrm{Ra}_{\perp}^{\text {conv }}$, defined by the divergence of $l_{e} \cdot{ }^{21}$

Config-2: to be able to analyze the fully established thermoconvective flow, especially for small $\mathrm{Ra}$, periodic boundary conditions have been implemented ${ }^{30}[$ Fig. 3(b)]. Furthermore, as at each time step, a transversal roll that leaves the computational domain is simultaneously sent to the inlet, config-2 allows us to determine $R a_{\perp}^{*}$; indeed, when being in the convectively unstable flow phase, the perturbations at the outlet are continuously reinjected at the entrance: a kind of forcing is maintained at the inlet of config- 2 .

The transversal roll wavelength, $\lambda$, is imposed by the aspect ratio $A$ of the domain. Computations with config-1 having shown that $\lambda \approx 1.95$, we take here $A=3.9$; thus, four transversal rolls may develop in config-2. Furthermore, $(\Delta x ; \Delta z)=(0.078 ; 0.05)$.

Config-3: taking advantage of the fact that, in config-2, the flow is periodic from the entrance to the outlet of the computational domain, it is possible to make the flow stationary with a frame of reference moving at the same velocity as the transversal rolls. Thus, config- 3 is the same as config-2 except that, using a very simple change of variable in Eqs. (1)-(3) the rolls are made stationary. Note that it has then been possible to take $\Delta t=0.01$, instead of $\Delta t=0.0005$, without losing accuracy.

To be complete, it can be added that, whatever the configuration, two initial conditions have been used: either an isothermal Poiseuille flow (at temperature $T_{c}$ ), or an already thermoconvective flow of transversal rolls; no hysteresis phenomenon has been observed with these conditions.

\section{RESULTS AND DISCUSSION}

All the results presented in the five following parts were obtained in the case of config-1, unless otherwise stated.

\section{A. Comparison with experiments - preliminary observations}

As a first result of the numerical simulation of the PBF, four particular flows are presented in Figs. 4, 5, 6 and 7. Figure 4 represents the time evolution of the experimental (part a) and computed (part b) vertical velocity component $W$, of the computed horizontal velocity component $U$ (part c) and of the space average Nusselt number $\mathrm{Nu}$ on the top and
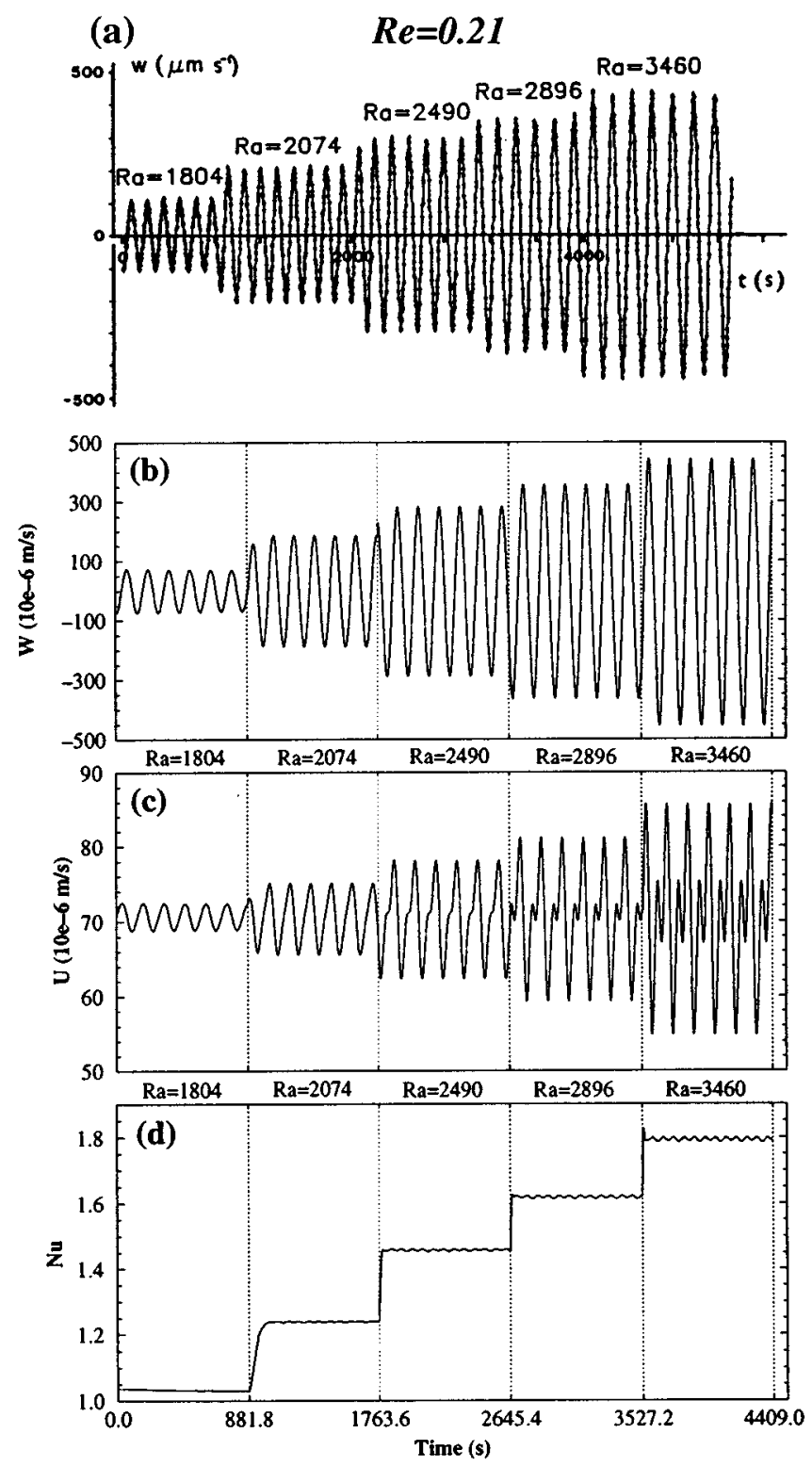

FIG. 4. Time evolution in the transversal roll phase, of the vertical $[W(t)]$ and horizontal $[U(t)]$ velocity components and of the space average Nusselt number $[\mathrm{Nu}(t)]$ for $\mathrm{Re}=0.21, \mathrm{Ra}=1804,2074,2490,2896$, and 3460, and for $\operatorname{Pr}=6.4$; (a) $W(t)$ at $(x, z)=(15.7,0.5)$ from the experiments of Ouazzani et al. $;^{14,16}(\mathrm{~b}),(\mathrm{c}),(\mathrm{d})$, respectively, $W(t), U(t)$, and $\mathrm{Nu}(t)$ at $(x, z)=(7.5$, $0.5)$ for the present numerical work (config-1).

bottom plates (part d), at $\mathrm{Re}=0.21$ for an increasing Rayleigh number. Figures 5, 6, and 7 represent the same quantities for an increasing $\mathrm{Re}$, respectively, for $\mathrm{Ra}=2420$, 2024, and 1804.

The computed velocity components, $U$ and $W$, are recorded at midheight of the channel $(z=0.5)$, at $x=7.5$. The numerical signals obtained by recording the dimensionless $W$ at each time step are presented dimensionally (in $\mu \mathrm{m} / \mathrm{s}$ ) in view of a comparison with the equivalent experimental data obtained by LDA in Refs. 14 and 16. These experimental recordings are realized at midheight, at $6.5 \mathrm{~cm}$ from the entrance of the channel, i.e., at $(x, z)=(15.7,0.5)$. For convenience, they are reproduced in Figs. 4(a), 5(a), 6(a), and 7(a) of this paper: these are, respectively, the Figs. 6 and 7 of Ref. 


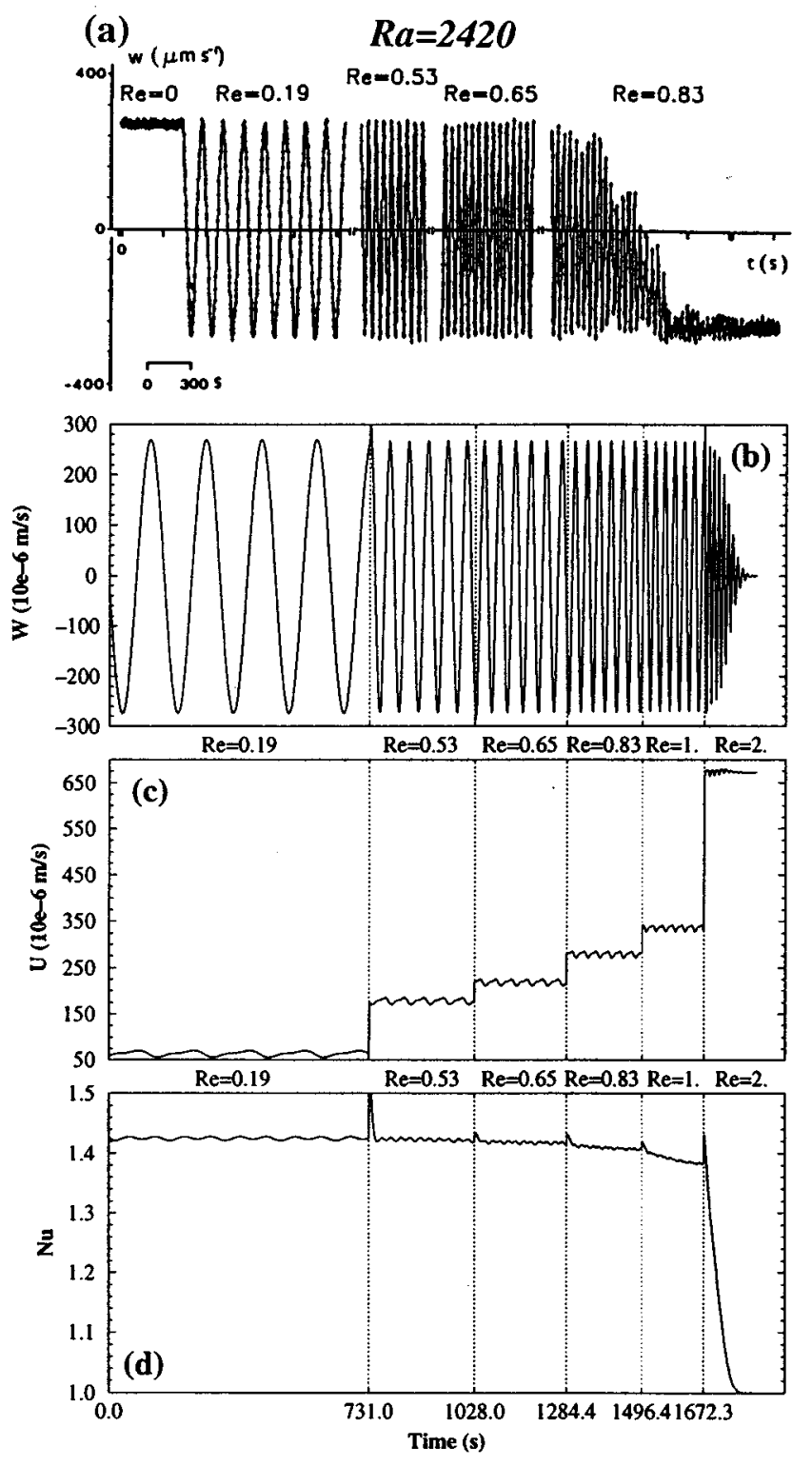

FIG. 5. The same as Fig. 4 for $\mathrm{Ra}=2420$ and $\mathrm{Re}=0.19,0.53,0.65$, and 0.83 (plus $\operatorname{Re}=1$ and $\mathrm{Re}=2$ for the numerical simulation); (a) for the experiments, ${ }^{14,16}$ transition to longitudinal rolls at $\mathrm{Re}=0.83$; (b),(c),(d) for the 2-D numerical simulations, transition to the Poiseuille flow at $\mathrm{Re}=2$.

16, Figs. 5-18 of Ref. 14, and Fig. 10 of Ref. 16. The time evolution of $U$ and $\mathrm{Nu}$ are only given for the numerical simulations since these quantities were not measured in the experimental available papers. $\mathrm{Nu}$ is defined as follows:

$$
\begin{aligned}
\mathrm{Nu}(t)= & \frac{1}{x_{2}-x_{1}} \int_{x_{1}}^{x_{2}} \frac{1}{2}\left[\left(\frac{\partial T}{\partial z}\right)_{z=0^{+}}(x, t)\right. \\
& \left.+\left(\frac{\partial T}{\partial z}\right)_{z=1^{-}}(x, t)\right] d x .
\end{aligned}
$$

The average over the length of the duct is taken between $x_{1}=4$ and $x_{2}=8.5$ in order to avoid the inlet and outlet zones in the evaluation of the mean. This is usually sufficient, except for extremely small Rayleigh numbers.

In Figs. 4(a) and 4(b), the sinusoidal behavior of $W$ around a zero mean value characterizes traveling transversal
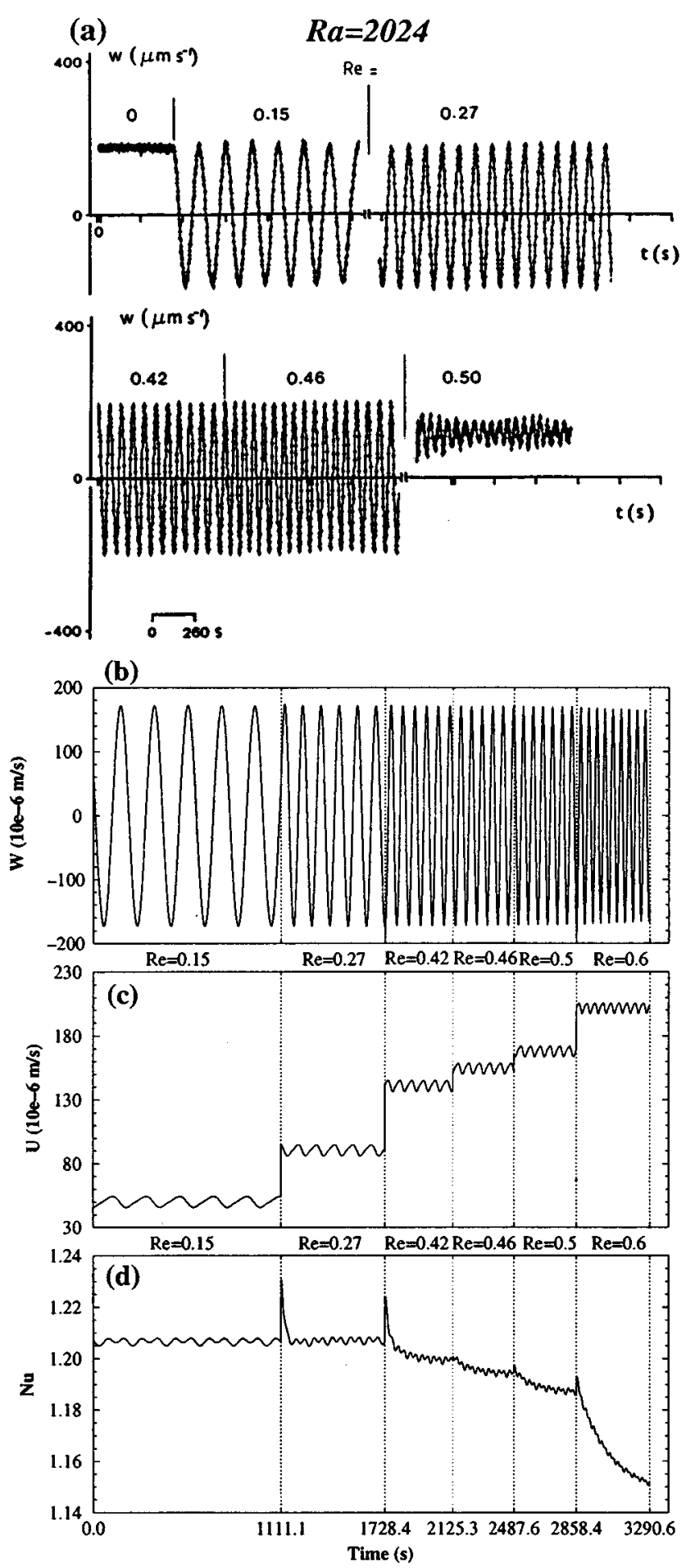

FIG. 6. The same as Fig. 4. for $\mathrm{Ra}=2024$ and $\mathrm{Re}=0.15,0.27,0.42,0.46$, and 0.5 (plus $\mathrm{Re}=0.6$ for the numerical simulation); (a) for the experiment, ${ }^{14}$ transition to "intermittent pattern" at $\mathrm{Re}=0.5$; (b),(c),(d) for the 2-D numerical simulation, persistence of the Poiseuille flow until Re $=0.6$.

rolls. $W_{\perp}^{\max }$ (the maximum vertical velocity component of the transversal rolls at midheight) increases with $\mathrm{Ra}$ and its square $\left(W_{\perp}^{\max }\right)^{2}$ is a linear function of Ra. The two signals, experimental and numerical, agree with each other, both in amplitude (except at $\mathrm{Ra}=1804$ ) and in frequency $f$. At Re $=0.21, f$ keeps constant whatever the Rayleigh number. It is approximately equal to $6.5 \times 10^{-3} \mathrm{~s}^{-1}$ in Fig. 4(a) and 


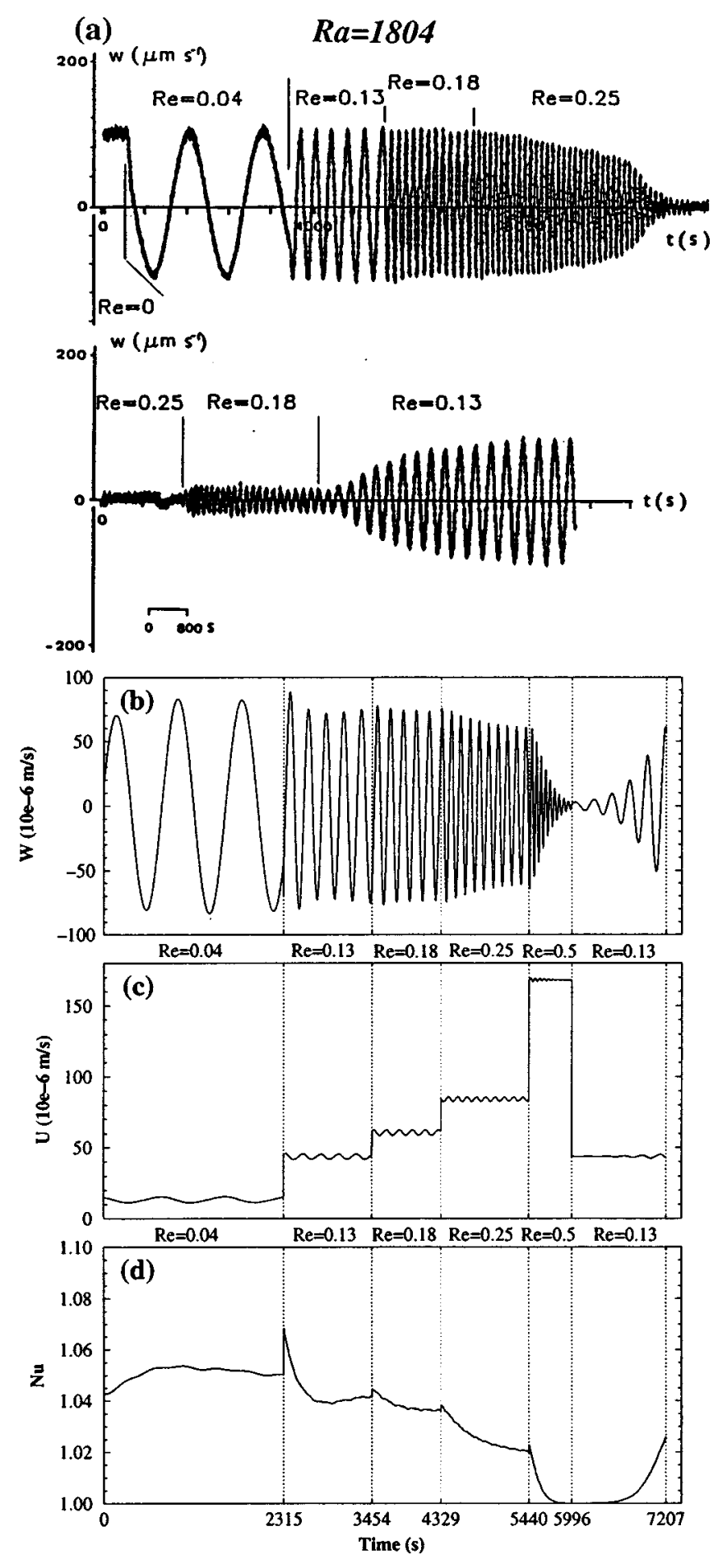

FIG. 7. The same as Fig. 4 for $\mathrm{Ra}=1804$ and $\mathrm{Re}=0.04,0.13,0.18$, and 0.25 (plus $\mathrm{Re}=0.5$ for the numerical simulation); (a) for the experiment, ${ }^{14,16}$ transition to the Poiseuille flow at $\operatorname{Re}=0.25$; (b),(c),(d) for the 2-D numerical simulation, transition to the Poiseuille flow at $\mathrm{Re}=0.5$.

$7.15 \times 10^{-3} \mathrm{~s}^{-1}$ in Fig. 4(b), that is to say a difference of $10 \%$.

Concerning the amplitude, Table II gives $W_{\perp}^{\max }$ for the experimental and numerical signals of Figs. 4, 5, 6, and 7. The maximum discrepancy between the two sets is less than $5 \%$, except at the smallest Rayleigh number, $\mathrm{Ra}=1804$, where it can reach as much as $40 \%$. This can partially be attributed to the difficulty of determining precisely the value of the Rayleigh number in the experiments (as already discussed in Ref. 23). Subsequently, as $W_{\perp}^{\max }$ is proportional to $\left(\mathrm{Ra} / \mathrm{Ra}_{\perp}^{\text {conv }}-1\right)^{1 / 2}$, the relative error on $W_{\perp}^{\max }$ increases when $\mathrm{Ra}$ tends to $\mathrm{Ra}_{\perp}^{\text {conv }}$. On the other hand, a part of the error can also be attributed to the position of the "measuring probe:" $x=15.7$ in the experiments and $x=7.5$ for the numerical simulations. At $\mathrm{Ra}=1804$, at $x=15.7$, the flow is fully developed, whereas, at $x=7.5$, we could still be in the entrance zone of the PBF, where the amplitude of $W$ has not yet reached its maximum.

In Fig. 4(c), the signal of $U$ becomes bichromatic, showing the fundamental frequency $f$ (the same as for $W$ ) and the first harmonic $2 f$. Their amplitudes increase with Ra. The harmonic $2 f$ has already been observed in the classical Rayleigh-Bénard convection. ${ }^{32,33}$ A detailed analysis of all our numerical results shows that the amplitudes of the two modes are independent of the Reynolds number, at least in the fully developed zone [this is, however, not clearly visible in Figs. 5(c), 6(c), and 7(c)]. In all cases, the mean value of $U$ corresponds exactly to the maximum Poiseuille velocity.

Figure 4(d) shows the increase of the Nusselt number with $\mathrm{Ra}$. The weak oscillations in the signal are due to the finite arbitrary width of the interval $\left[x_{1}, x_{2}\right]$ in the computation of $\mathrm{Nu}$ [cf. Eq. (4)], which do not necessarily contain a finite number of rolls. Consequently, we will note $\langle\mathrm{Nu}\rangle$ the time average Nusselt number over these oscillations:

$$
\langle\mathrm{Nu}\rangle=\frac{1}{t_{2}-t_{1}} \int_{t_{1}}^{t_{2}} \mathrm{Nu}(t) d t,
$$

where the time interval $\left(t_{2}-t_{1}\right)$ excludes the small initial transient phase when increasing the Rayleigh number.

In each of the Figs. 5, 6, and 7, the Rayleigh number is constant. If the Reynolds number is small enough (for the values listed in Table II), stabilized transversal roll flows are observed: $W_{\perp}^{\max }$ (and $\langle\mathrm{Nu}\rangle$ in a lesser extent) keeps constant with Re (cf. Table II). In addition, the roll frequency increases linearly with Re (shown in detail later). In Fig. 5(a), when Re increases, the flow undergoes experimentally a transition to longitudinal rolls $\left(\mathrm{Ra}>\mathrm{Ra}_{\|}^{*}\right)$, characterized by a nonzero and constant vertical velocity component, at $\mathrm{Ra}$ $=2420$ and $\operatorname{Re}=0.83$; in the 2-D numerical simulation, the transition is to the Poiseuille flow, at $\mathrm{Re}=2$, characterized by $W=0$ and a constant $U$ signal together with a Nusselt number equal to 1 [Figs. 5(b)-5(d)]. In Fig. 6, at a lower Rayleigh number $(\mathrm{Ra}=2024)$, the scenario remains the same, except for the so-called "intermittent pattern", 17 in the experimental signal at $\mathrm{Re}=0.5$. In Fig. 7, for a still lower $\mathrm{Ra}$ $(\mathrm{Ra}=1804)$, a transition to the Poiseuille flow is observed in the two cases since now $\mathrm{Ra}<\mathrm{Ra}_{\|}^{*}$; it occurs between $\mathrm{Re}$ $=0.18$ and $\operatorname{Re}=0.25$ in the experiments, and between $\operatorname{Re}$ $=0.25$ and $\mathrm{Re}=0.5$ in the numerical simulations (a more precise determination yields $\mathrm{Re}=0.36$ ). When the Reynolds number is reduced to the previous value $\mathrm{Re}=0.13$, transversal rolls with the same amplitude and the same frequency are restored.

As already mentioned before, an aspect ratio $L / h=10$ computational domain is unsufficient to get fully established flows at the lowest $\mathrm{Ra}(\mathrm{Ra}=1804$ in Table II). So, in the 
TABLE II. Comparison of the maximum vertical velocity component during the transversal roll phase, as a function of Ra and Re, obtained experimentally by Ouazzani et al. ${ }^{14,16}$ and numerically in the present work.

\begin{tabular}{lccccc}
\hline \hline & & & $\begin{array}{c}W_{\perp}^{\max }(\mu \mathrm{m} / \mathrm{s}) \text { at } x=15.7 \\
\text { Experimental work }{ }^{14,16}\end{array}$ & $\begin{array}{c}W_{\perp}^{\max }(\mu \mathrm{m} / \mathrm{s}) \text { at } x=7.5 \\
\text { Present }\end{array}$ & $\begin{array}{c}\text { Discrepancy } \\
(\%)\end{array}$ \\
\hline Fig. 4 & Ra & $\operatorname{Re}$ & 106 & 71 & 39.5 \\
& 1804 & 0.21 & 197 & 189 & 4.1 \\
& 2074 & & 292 & 289 & 1.0 \\
& 2490 & & 357 & 365 & 2.2 \\
& 2896 & & 432 & 455 & 5.0 \\
Fig. 5 & 3460 & & 273 & 274 & 0.4 \\
& 2420 & 0.19 & 273 & 273 & 0.0 \\
& & 0.53 & 273 & 273 & 0.0 \\
Fig. 6 & 2024 & 0.65 & 180 & 174 & 3.4 \\
& & 0.15 & 180 & 174 & 3.4 \\
Fig. 7 & 1804 & 0.04 & 180 & 173 & 4.0 \\
& & 0.13 & 106 & 74 & 32.4 \\
\hline \hline
\end{tabular}

subsequent paragraphs and figures, for $\mathrm{Ra}=1804$ and 1836, $L / h=20$ is always used and results in better agreement with the experimental values of Table II.

\section{B. Space development of the transversal rolls}

As was already stated in the Introduction, the observed critical Rayleigh number compares with $\mathrm{Ra}_{\perp}^{\text {conv }}$ and not with $\mathrm{Ra}_{\perp}^{*}$. In this paragraph, we want to numerically recover, for $\operatorname{Pr}=6.4$, previous results obtained experimentally and theoretically concerning $\mathrm{Ra}_{\perp}^{\text {conv }}$; eventually, we also want to show that the numerical tool allows us to give results at high Rayleigh numbers, where a Ginzburg-Landau-type approach certainly fails to produce accurate results. Therefore, in Figs. 8 and 9, at different $\mathrm{Ra}$ and $\mathrm{Re}$, we present numerous stationary envelopes of the maximum vertical velocity component along the axis of the channel. More precisely, the plots of the figures give $W_{\max }$ as a function of $x$, where

$$
W_{\max }(x)=\max _{t \in\left[t_{1}, t_{2}\right]}\left(\max _{z \in[0,1]} W(x, z, t)\right),
$$

with $t_{2}>t_{1}$ and $t_{1}>t_{t}$, where $t_{t}$ is the time marking the end of the transient flow phase.

As an example, let us focus on the graph drawn at $\mathrm{Ra}$ $=1836$ for which the computational domain has been extended to $L / h=20$ in order to avoid the effect of the OBC, clearly important at small $\mathrm{Ra}$ and $\mathrm{Re}$. The saturation amplitude $W_{s}$, defined by

$$
W_{s}=\max _{x \in\left[x_{\text {in }}, x_{\text {out }}\right]} W_{\max }(x),
$$

is equal to $102 \mu \mathrm{m} / \mathrm{s}$. The characteristic growth length $l_{e}$ is defined by $W_{\max }\left(l_{e}\right)=W_{s} / 2$. For each Reynolds number, $l_{e}$ has been determined from the plots of Fig. 8, and is given in Fig. 10. Its divergence at $\operatorname{Re} \approx 0.44$ is clearly seen to coincide with the result of the amplitude equation ${ }^{23}$ and to agree fairly well with the experiments (dashed line). The results at Ra $=1804$ and 2024 are also reported; of course, the divergence of $l_{e}$ is found earlier $(\operatorname{Re} \approx 0.36)$ at $\mathrm{Ra}=1804$ and later $(\operatorname{Re}$ $\approx 0.8)$ at $\mathrm{Ra} \approx 2024$. As a matter of fact, the procedure can be repeated for all the Rayleigh numbers presented in Fig. 8 and the divergence of $l_{e}$ appears at higher Reynolds numbers when Ra increases (not shown in Fig. 10). The Poiseuille
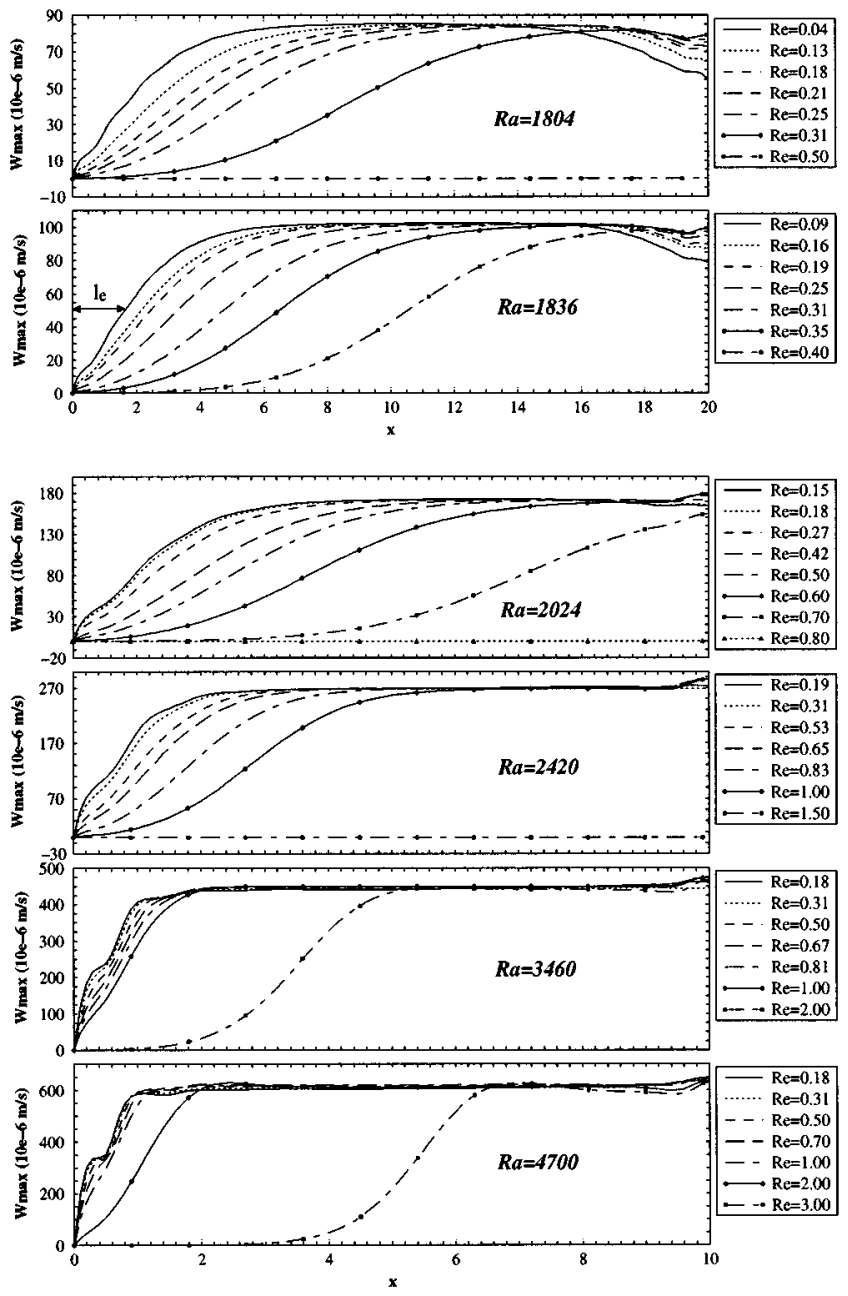

FIG. 8. For fixed Ra and several Re, stationary envelopes representing the space evolution of the maximum vertical velocity component along the axis of the channel, from the inlet to the outlet of config-1 domain; for $\mathrm{Ra}=1804$ and $\mathrm{Ra}=1836, A=L / h=20$; for $\mathrm{Ra}=2024,2420,3460$, and $4700, A=10$. 

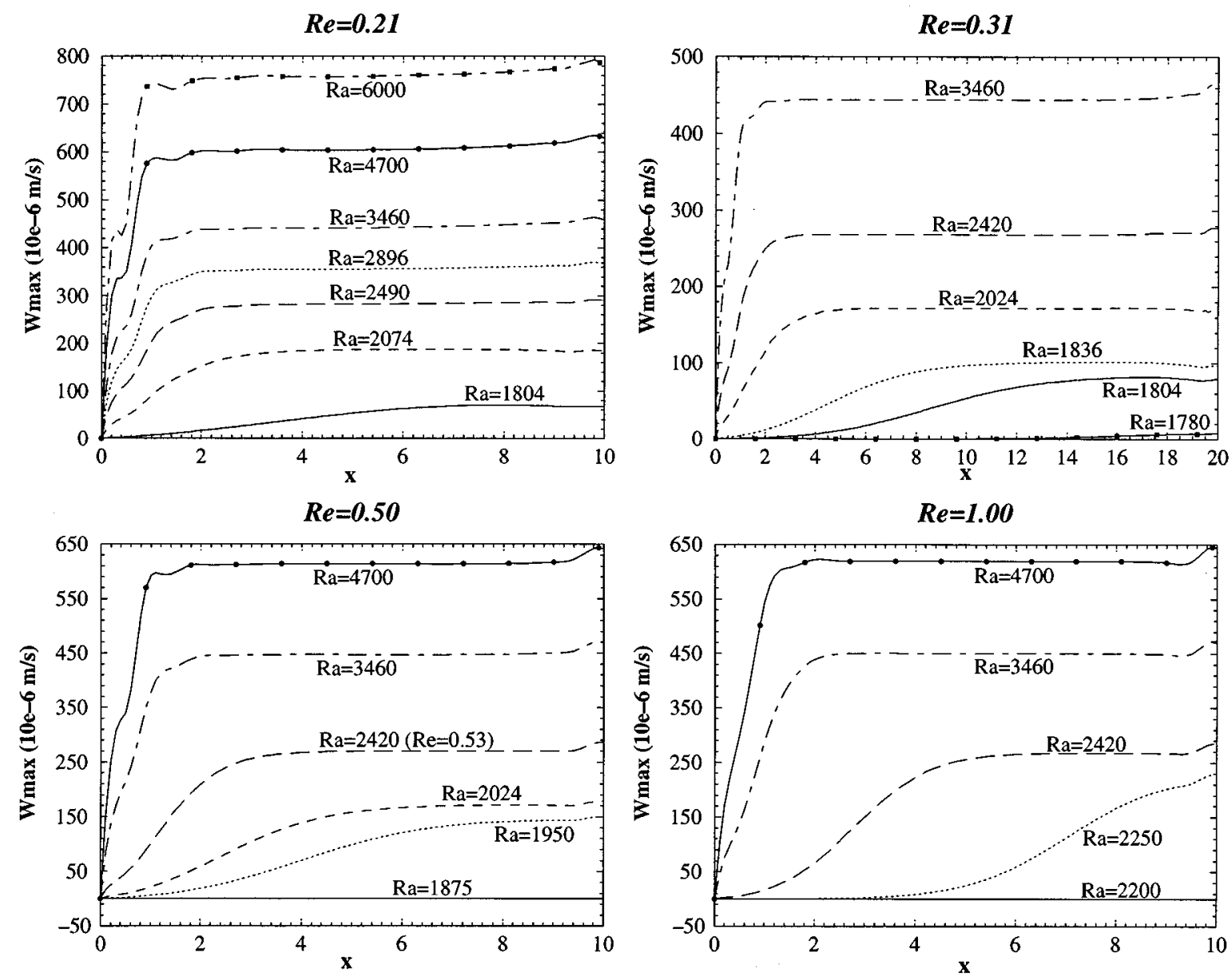

FIG. 9. For fixed Re and several Ra, stationary envelopes representing the space evolution of the maximum vertical velocity component along the axis of the channel, from the inlet to the outlet of config-1 domain; for $\operatorname{Re}=0.21,0.5$, and $1, A=L / h=10$; for $\operatorname{Re}=0.31, A=20$.

flow is reached when $W_{\max }(x)=0$ for all $x \in[0, L / h]$; it is only shown for $(\mathrm{Ra}, \mathrm{Re})=(1804,0.5),(2024,0.8)$, and $(2420,1.5)$ in Fig. 8 , and for $(\mathrm{Re}, \mathrm{Ra})=(0.5,1875)$ and $(1$, 2200) in Fig. 9.

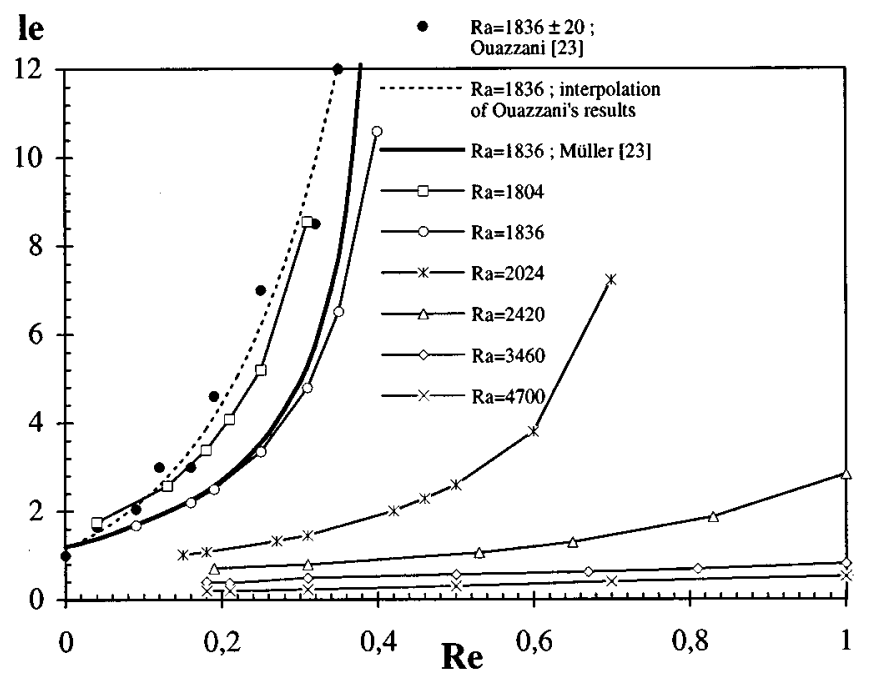

FIG. 10. Characteristic growth length over which the vertical velocity envelope of the transversal rolls, $W_{\max }(x)$, increases from the inlet to half its value of saturation $\left(W_{s} / 2\right)$, as a function of $\mathrm{Re}$; at $\mathrm{Ra}=1836$, comparison with Ouazzani's experiment ${ }^{23}$ and with a result obtained from the amplitude equation theory by Müller. ${ }^{23}$
In Fig. 11, the saturation amplitude of the vertical velocity component, $W_{s}$, is shown to be independent of the Reynolds number (as it should be), and is compared with the experiments of Ouazzani et al. ${ }^{14,16}$ The maximum discrepancy between the results of the two studies is at most 14 $\mu \mathrm{m} / \mathrm{s}$.

\section{Stability diagram in (Ra-Re) plane}

Figure 12 presents the stability diagram in the $\mathrm{Ra}-\mathrm{Re}$ plane. The results of the linear ${ }^{12}$ and of the convective $\mathrm{e}^{21,23,24}$ stability theory, together with the experimental results of Ouazzani et al. ${ }^{14,16}$ are superimposed to the results of the present numerical work. The theoretical results apply to 2-D flows, whereas the experimental results are 3-D; consequently, the experiments allow more flow configurations and transitions.

Numerically, the nature of each point of the diagram, Poiseuille flow, or transversal rolls, is determined by following the evolution of a given transversal roll flow as initial conditions and updating Re and/or Ra. In some cases, we have verified that the choice of the initial conditions do not lead to hysteresis effects: the same transversal roll flow is computed starting with a conductive solution of the PBF and adding a small sinusoidal perturbation on $W$ equal to $5 \%$ of the final amplitude of the rolls. In practice, very long CPU times are needed to compute the points near the transition 


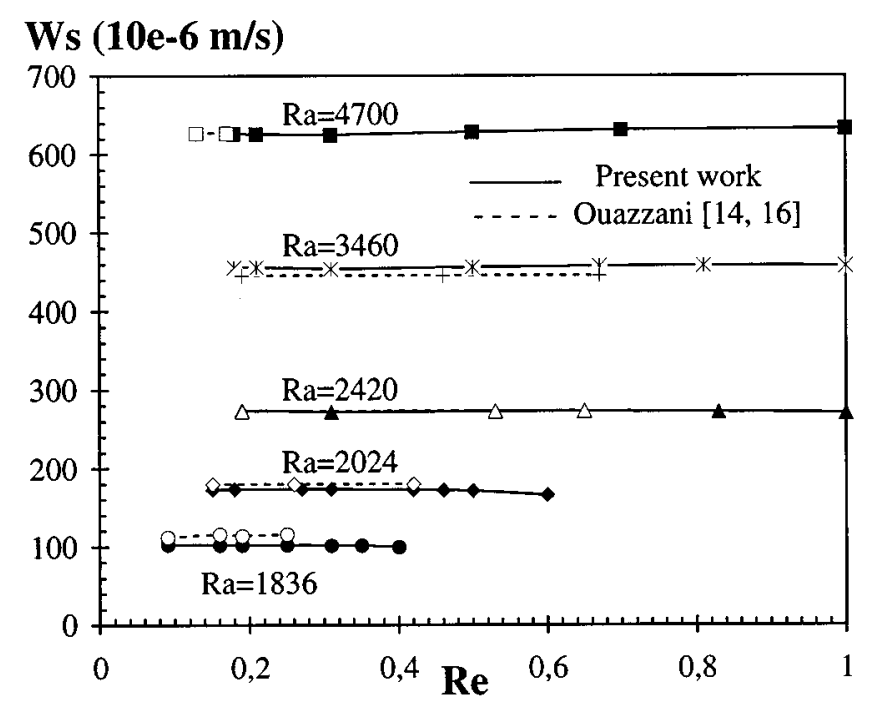

FIG. 11. Saturation amplitude of the vertical velocity component as a function of Re, for the experiments of Ouazzani et al. ${ }^{14,16}$ and for the present numerical work.

curve. Consequently, only 19 points have been computed to determine its position. Furthermore, to save computational time, all the computations, even for the smallest Rayleigh numbers, have been realized with config-1 for $L / h=10$. However, a few times, $L / h=20$ has been used in order to assess the presence of the transversal rolls; for example, in Fig. 9, at $\mathrm{Re}=0.31$ and $\mathrm{Ra}=1780$, i.e. close to $\mathrm{Ra}_{\perp}^{\text {conv }}$, the instability seems to develop only after $x=10$. Nevertheless, this remark is a function of the criterion used to determine the nature of the flow; in this study, it is considered that the transversal rolls disappear for the benefit of the Poiseuille flow when the following criterion is verified:

$$
\left\{\begin{array}{l}
\forall x \in[0,10], \quad W_{\max }(x)<0.1 \quad \mu \mathrm{m} / \mathrm{s}, \\
\text { and } \\
\langle\mathrm{Nu}\rangle-1<10^{-6} .
\end{array}\right.
$$

Figure 12 shows that the numerical results coincide quite well with the results of the amplitude equation; therefore, the 2-D numerical simulations of the PBF with config-1 satisfies the convective stability criterion, not the linear one.

Experimentally, the stability diagram of the 3-D flow is much more complex. Ouazzani et al. ${ }^{14,16}$ have identified five regions (cf. Fig. 12): for the small Rayleigh numbers (zone I), the Poiseuille flow keeps stable; for the bigger Rayleigh numbers the flow is thermoconvective: transversal rolls are always observed in II and longitudinal rolls in III; between these two zones, in IV, there are either transversal or longitudinal rolls, depending on the initial conditions; and, in the small zone $\mathrm{V}$, the flow structure shows an intermittent character for which the vertical velocity $W$ oscillates around a nonzero mean value. ${ }^{16,17}$ Except for the smallest Reynolds

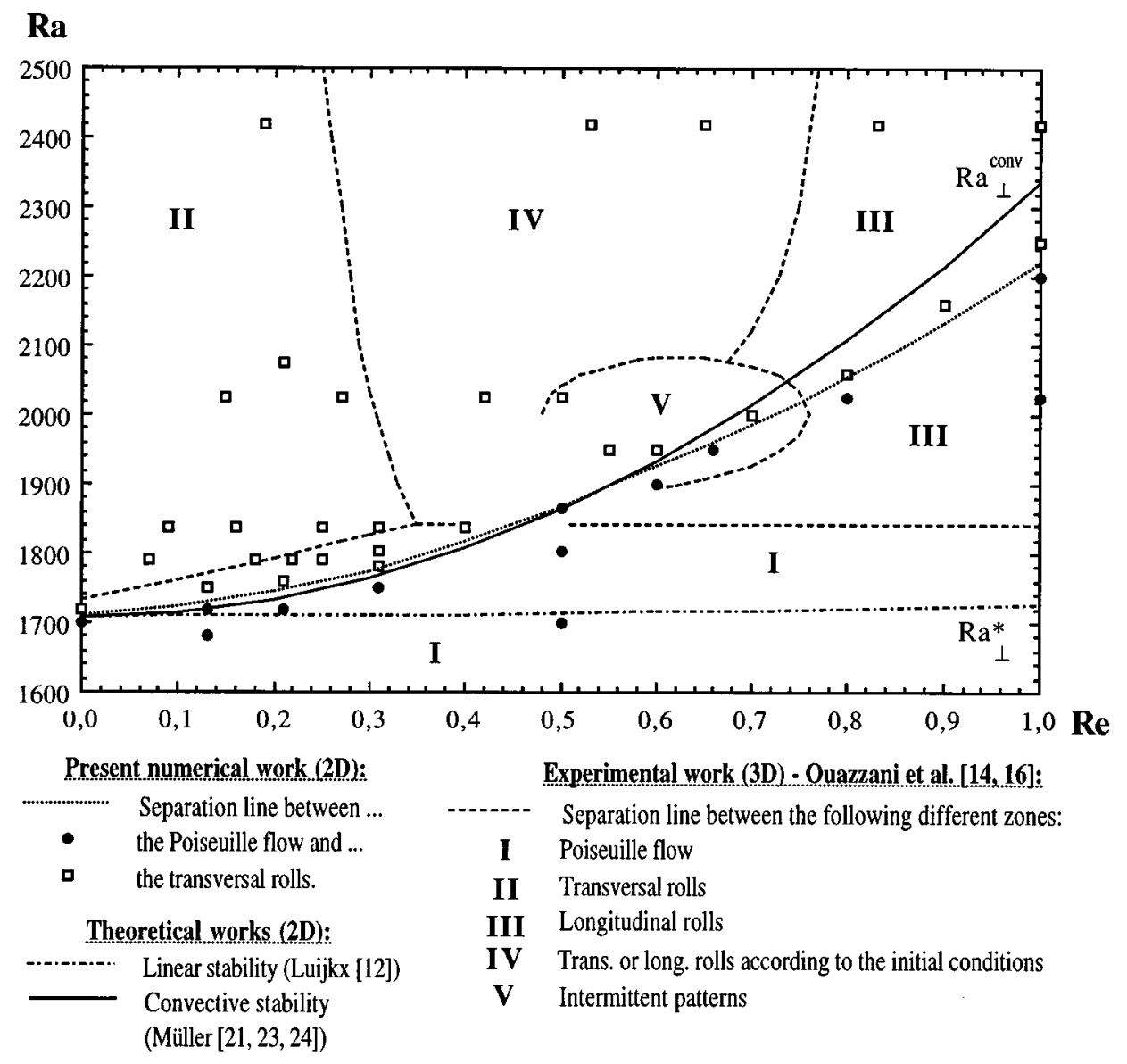

FIG. 12. Stability diagram of the different configurations encountered in the PBF with $\operatorname{Pr}=6.4$. The numerical results of the present work are obtained with config-1; other already published results are superimposed to the numerical solution. 


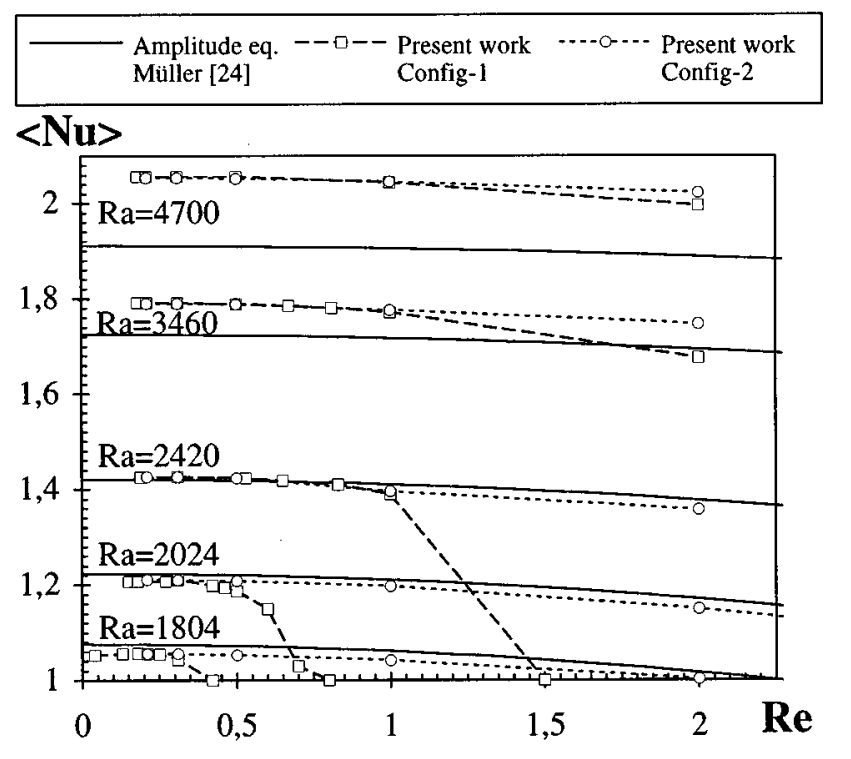

FIG. 13. Space and time average Nusselt number as a function of Re and $\mathrm{Ra}$; comparison of the numerical solutions obtained on config-1 and config- 2 with the linear stability result obtained by Müller. ${ }^{24}$

numbers $\left(<\mathrm{Re}^{*} \approx 0.3\right)$, it is difficult to compare the experimental stability diagram with the others: a 3-D numerical simulation would be necessary. Nevertheless, two 3-D numerical studies of the PBF can be pointed out: in Ref. 15, a zone IV is observed for a flow in a transversal aspect ratio 2 duct and for $\operatorname{Pr}=0.7$ (even though this result seems to be linked with a numerical artefact: the choice of $\Delta t$ ); in Ref. 18 , with $B=l / h=4.1$ and $\operatorname{Pr}=530$ (silicon oil), Schröder and Bühler show flow configurations where transversal and longitudinal rolls superimpose; these solutions could be favorably compared with certain experimental signals obtained in region $\mathrm{V}$ by Ouazzani et al. ${ }^{14,17}$

\section{Nusselt number}

In Fig. 13, the space and time average Nusselt number $\langle\mathrm{Nu}\rangle$ is plotted as a function of Re for several values of Ra. The results of the numerical simulations, computed with config- 1 and config-2, are compared with those obtained by Müller $^{24}$ and valid for a 2-D flow. From the amplitude equation, Müller proposes the following formula to compute the horizontally average Nusselt number:

$$
\langle\mathrm{Nu}\rangle=1+\frac{1}{\gamma} \frac{\mathrm{Ra}-\mathrm{Ra}_{\perp}^{*}}{\mathrm{Ra}},
$$

where, for $\operatorname{Pr}=6.4, \gamma=0,699$, i.e. $1 / \gamma=1.4306$ (see Ref. 23).

On the other hand, Schlüter et al. $^{34}$ give a formula valid on a linear domain for pure free convection and an infinite roll pattern between two horizontal plates:

$$
\langle\mathrm{Nu}\rangle=1+K \frac{\mathrm{Ra}-1708}{\mathrm{Ra}},
$$

where

$$
\begin{aligned}
K & =\frac{1}{0.69942-0.00472 / \operatorname{Pr}+0.00832 / \mathrm{Pr}^{2}} \\
& =1.4308 \text { for } \operatorname{Pr}=6.4 .
\end{aligned}
$$

Therefore, Müller's formula (9) is nothing else but the Schlüter et al. formula (10) in which 1708 , is replaced by $\mathrm{Ra}_{\perp}^{*}$; thus, $\langle\mathrm{Nu}\rangle$ in (9) is a function of $\mathrm{Re}$ through $\mathrm{Ra}_{\perp}^{*}$ and it is valid only for small Rayleigh numbers.

For the numerical computations with config-1 at $\mathrm{Ra}$ $=1804$, the Nusselt number is averaged from $x_{1}=10$ to $x_{2}=18$ [cf. (4)] because $A=20$; in the other cases, $A=10$ and the average is taken, as said before, from $x_{1}=4$ to $x_{2}=8.5$. For config-2, $A=3.9$ and $\langle\mathrm{Nu}\rangle$ is averaged from $x_{1}=0.2$ to $x_{2}=3.7$.

In the three different studies, the Nusselt number decreases with Re. It tends to $\langle\mathrm{Nu}\rangle=1$, corresponding to the conductive Poiseuille flow. With config-1, this limit is reached when crossing the $\mathrm{Ra}_{\perp}^{\text {conv }}$ curve, as it can be seen at $\mathrm{Ra}=1804,2024$, and 2420. Obviously, $\langle\mathrm{Nu}\rangle$ obtained with config-1 and config- 2 coincide very well whatever Ra, for small values of $\mathrm{Re}$, i.e., for the cases where, in config- 1 , the transversal roll amplitude has reached its stationary value between $x_{1}$ and $x_{2}$.

For config-2, $\langle\mathrm{Nu}\rangle$ decreases slightly with $\mathrm{Re}$, until the value $\langle\mathrm{Nu}\rangle=1$ is reached when crossing the neutral critical curve $\mathrm{Ra}_{\perp}^{*}$. It has been computed earlier ${ }^{12}$ that $\mathrm{Ra}_{\perp}^{*}=1804$ at $\operatorname{Re}=2.27$ and $\mathrm{Ra}_{\perp}^{*}=2024$ at $\mathrm{Re}=4.12$. We find here that $\langle\mathrm{Nu}\rangle$ drops to 1 at $\mathrm{Ra}=1804$ when $\mathrm{Re} \approx 2.05$ instead of 2.27 (see the lower curve of Fig. 13), and at $\mathrm{Ra}=2024$ when $\mathrm{Re}$ $\approx 4.2$ instead of 4.12 (not shown in Fig. 13). The Nusselt number computed with Eq. (9) is in agreement with the numerically found values, at least at small Rayleigh numbers $(\mathrm{Ra} \leqslant 2420)$. At higher $\mathrm{Ra}$, Eq. (9) cannot remain a good approximation.

\section{E. Transversal roll frequency, wavelength, and velocity}

In Fig. 14, the transversal roll frequency $f$, computed with config-1, is plotted as a function of Re, for several Rayleigh numbers. The frequency has been determined from the same type of signals as those presented in Figs. 4-7. For a fixed Rayleigh number, $f$ is a linear function of Re. It has been verified that $f$ does not vary with $x$, as long as $x<x_{\text {out }}$. The numerical results are in good agreement with the experimental results of Ouazzani. ${ }^{14}$ The fact that all the curves do not pass through the origin is attributed to the outlet $\mathrm{OBC}$, a fact already observed and discussed in Ref. 18. With the use of periodic boundary conditions (config-2), all the curves do pass through the origin. In contradistinction with Ref. 18 (a study realized at a totally different Prandtl number $\operatorname{Pr}=530$ ), in which the authors found that $f$ decreases when $\mathrm{Ra}$ increases whatever $\mathrm{Re}$, we find in our simulations that this is only the case for $\operatorname{Re}<0.26$; beyond, $f$ increases slightly when $\mathrm{Ra}$ increases. This is due to the small mismatches of the wavelength in the two studies, conducted at very different Prandtl numbers.

The dimensionless wavelength, $\lambda$, is plotted in Fig. 15. Here, $\lambda$ is computed averaging from the signals of $U, W$, and 


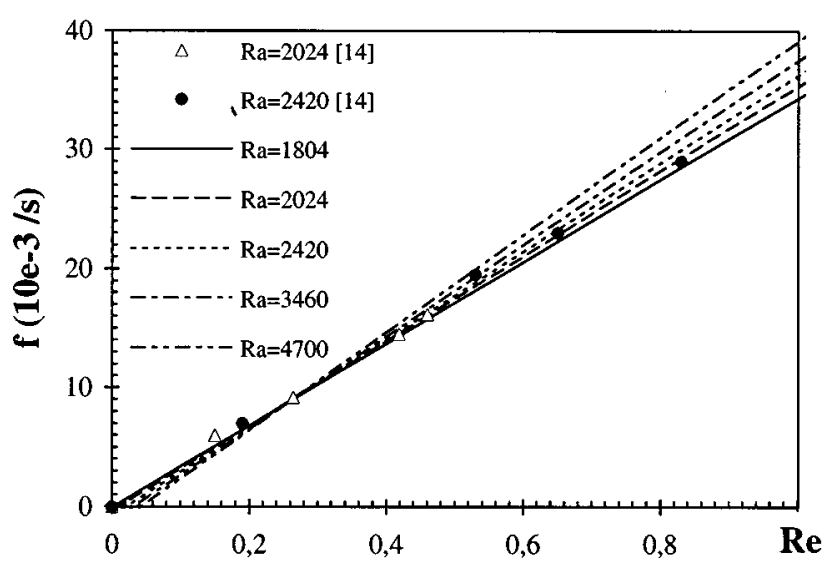

FIG. 14. Transversal roll frequency, computed on config-1, as a function of $\mathrm{Re}$ and $\mathrm{Ra}$ and for $\mathrm{Pr}=6.4$; comparison with Ouazzani's experiment ${ }^{14}$ at $\mathrm{Ra}=2024$ and 2420 .

$T$ recorded for $x_{1} \leqslant x \leqslant x_{2}$, at eight different time steps for $t \geqslant t_{t}$. From a single signal, when $A=10, \Delta x=0.1$, and $x_{2}-x_{1}=4.5, \lambda$ is approximately evaluated within \pm 0.05 . But, by multiplying the number of signals (both for $U, W$, and $T$ at eight different times, i.e. 24 signals), the error on $\lambda$ for each point of Fig. 15 is estimated to be less than 0.015 (the error bars are drawn only for a few points). So, in spite of an inaccuracy linked to the mesh size, $\lambda$ is shown to weakly decrease when Re and Ra increases (except for the smallest Reynolds numbers $R e \leqslant 0.3$ ). This is in good qualitative agreement with the result of the 2-D numerical simulation by Müller et $a l .{ }^{21}$ at $\operatorname{Pr}=1$, and with those of Schröder and Bühler, ${ }^{18}$ who found the same tendency for $\lambda$, even at $\operatorname{Pr}=530$.

Figure 16 presents $V r / U^{\circ}=\lambda / \tau=\left[h^{2} /(\nu \operatorname{Re})\right] f \lambda$ as a function of $\mathrm{Ra}$ (where $\tau$ is the dimensionless time period, $\lambda$ the dimensionless wavelength plotted in Fig. 15, but $f$ is the frequency in $\mathrm{s}^{-1}$ plotted in Fig. 14). As already said in the Introduction, the four plotted curves, corresponding to Ouazzani's experiment, ${ }^{14,16}$ Müller's 2-D theory, ${ }^{24}$ and to the present numerical results obtained with config-1 and config-3, decrease linearly with $\mathrm{Ra}$ and are independent of Re. The three 2-D studies give nearly the same curves, and

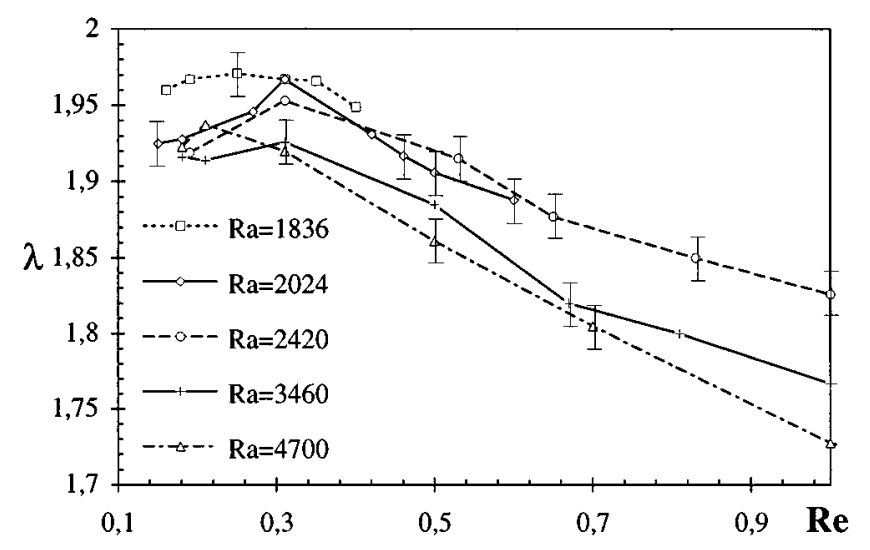

FIG. 15. Transversal roll dimensionless wavelength (with error bars drawn for a few points), computed on config-1, as a function of Re and $\mathrm{Ra}$ and for $\operatorname{Pr}=6.4$.

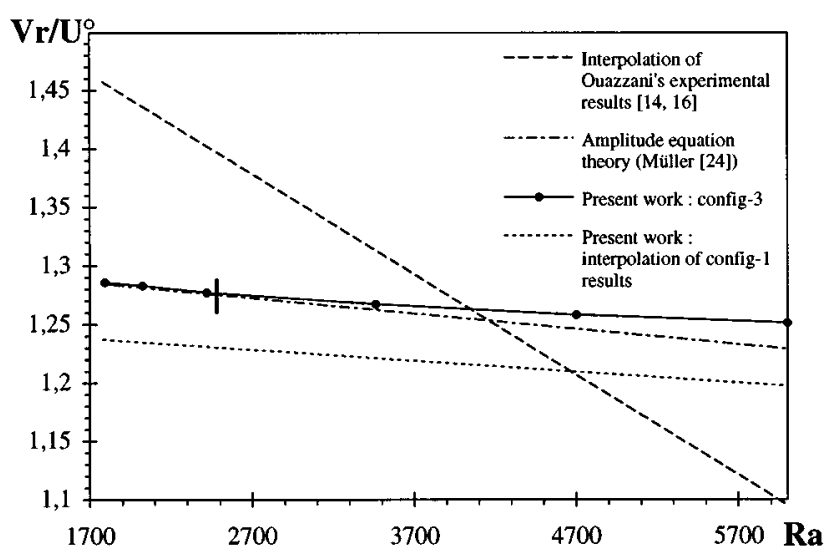

FIG. 16. Ratio of the transversal roll velocity, $V r$, to the average velocity of the flow, $U^{\circ}$, as a function of $\mathrm{Ra}$ and for $\mathrm{Pr}=6.4$; comparison of the numerical solutions obtained on config- 1 and config- 3 with the experimental results of Ouazzani et al. ${ }^{14,16}$ and with Müller's theoretical result ${ }^{24}$ (the limit of validity of this theory, $\mathrm{Ra} \approx 2500$, is indicated by a vertical line). All the curves are independent of Re.

the linear stability result obtained by Luijkx ${ }^{12}$ in the case of infinite lateral extension ducts $\left(V r / U^{\circ}=1.29\right.$ interpolated for $\operatorname{Pr}=6.4)$ is exactly reproduced by the amplitude equation theory and by the present simulations with config-3. Ouazzani's 3-D result is also in good agreement with the linear stability theory, ${ }^{12}$ where we estimate $V r / U^{\circ} \approx 1.5$ at the critical point for $B=l / h=3.6$. Therefore, the difference between $\mathrm{Vr} / U^{\circ}=1.29$ and 1.5 is clearly due to the lateral confinement of the fluid. The slope of the only available experimental curve has never been theoretically verified yet; a 3-D numerical simulation would be necessary.

For config-1, the curve of Fig. 16 is the result of the linear interpolation of $V r / U^{\circ}$ computed from $f$ and $\lambda$; since $f$ increases with $\operatorname{Ra}$ (when $\operatorname{Re} \geqslant 0.26$ ), the decrease of $V r / U^{\circ}$ is due to the faster decrease of $\lambda$. The Re independence of $V r / U^{\circ}$ is determined within \pm 0.03 . The line equation is $V r / U^{\circ}=1.254-0.95 \times 10^{-5} \mathrm{Ra}$.

Müller's curve and that of the simulation with config-3 merge into one single straight line in the validity domain of the Ginzburg-Landau equation: for $\mathrm{Ra}<2500, \mathrm{Vr} / U^{\circ}$ $=1.308-1.32 \times 10^{-5} \mathrm{Ra}$. The curve with config- 2 (not drawn in the graph) is parallel and at 0.02 units below the curve of config-3. With the periodic boundary conditions, since $\lambda$ is constant, $V r / U^{\circ}$ is more precisely determined than when $\lambda$ varies; the $R e$ independence is once more verified, within \pm 0.01 for config- 2 and within \pm 0.002 for config-3. Furthermore, $V r / U^{\circ}$ is not modified when the longitudinal aspect ratio $A$ of these two configurations varies between 3.5 and 4.5: the roll frequency adjusts itself to the imposed wavelength so that the roll velocity remains the same. Finally, let us note that Hasnaoui et al. ${ }^{4}$ have found a result very close to our finding for the roll velocity, also using periodic boundary conditions similar to config-2; their line equation, for $\operatorname{Pr}=7$, is $V r / U^{\circ} \approx 1.284-0.90 \times 10^{-5} \mathrm{Ra}$.

\section{CONCLUSION}

In this study, numerous results characterizing the PBF, in a wide range of the dimensionless parameters $\mathrm{Ra}$ and $\mathrm{Re}$, 
have been presented in detail; each time possible, they are validated thanks to quantitative comparisons with the already published experimental and theoretical works.

For the transversal roll configuration, the time evolution of the vertical velocity and the frequency have been shown to be in a very good agreement with the experiments of Ouazzani et al., ${ }^{14,16}$ as soon as $\mathrm{Ra}>2000$. The theoretical results of Müller et al., ${ }^{21,23,24}$ based on the Ginzburg-Landau amplitude equation and valid for $\mathrm{Ra}<2500$, are also well reproduced: particularly, the results concerning the absolute instability-convective instability transition between the transversal rolls and the Poiseuille flow, the characteristic growth length $l_{e}$, the variation of the velocity $V r / U^{\circ}$ with $\mathrm{Ra}$, and the variation of the Nusselt number with Re. However, a 3-D numerical simulation seems to be necessary in view of an explanation of the experimental slope concerning $\mathrm{Vr} / U^{\circ}$, and, obviously, concerning the reproduction of the stability map and the numerous varieties of flow patterns experimentally observed (longitudinal rolls, intermittent patterns,...).

Finally, it has been shown that the simulations with a computational configuration with OBC at the outlet (config-1 type) give the convective stability curve $\mathrm{Ra}_{\perp}^{\text {conv }}$, whereas a config-2 type configuration (with periodic boundary conditions) simulates the linear stability curve $\mathrm{Ra}_{\perp}^{*}$.

\section{ACKNOWLEDGMENTS}

This work was supported by CNRS/CGRI-FNRS (Grants EB/EUR-94/No. 41 and EB/EUR-95/No. 123). The numerical simulations were performed on the IBM-SP2 computer of the CNUSC (South University National Center of Computation). The authors would like to thank the staff of the CNUSC for his availability concerning the technical support and the training.

${ }^{1}$ H. Bénard and D. Avsec, "Travaux récents sur les tourbillons cellulaires et les tourbillons en bandes; applications à l'astrophysique et à la météorologie," J. Phys. Rad. 9, 468 (1938).

${ }^{2}$ D. Brunt, "Experimental cloud formation," Compendium of Meteorology (American Meteorological Society, Boston, 1951), pp. 1255-1262.

${ }^{3}$ M. E. Braaten and S. V. Patankar, "Analysis of laminar mixed convection in shrouded arrays of heated rectangular blocks," Int. J. Heat Mass Transfer 28, 1699 (1985).

${ }^{4}$ M. Hasnaoui, E. Bilgen, P. Vasseur, and L. Robillard, "Mixed convective heat transfer in a horizontal channel heated periodically from below," Num. Heat Transfer, Part A. 20, 297 (1991).

${ }^{5} \mathrm{G}$. Evans and R. Greif, "A study of traveling wave instabilities in a horizontal channel flow with applications to chemical vapor deposition," Int. J. Heat Mass Transfer 32, 895 (1989).

${ }^{6} \mathrm{G}$. Evans and R. Greif, "Unsteady three-dimensional mixed convection in a heated horizontal channel with applications to chemical vapor deposition,' Int. J. Heat Mass Transfer 34, 2039 (1991).

${ }^{7}$ G. Evans and R. Greif, "Thermally unstable convection with applications to chemical vapor deposition channel reactors," Int. J. Heat Mass Transfer 36, 2769 (1993).

${ }^{8}$ H. K. Moffat and K. F. Jensen, "Complex flow phenomena in MOCVD reactors-1. Horizontal reactors,', J. Cryst. Growth 77, 108 (1986).

${ }^{9}$ K. C. Chiu and F. Rosenberger, "Mixed convection between horizontal plates-1. Entrance effects," Int. J. Heat Mass Transfer 30, 1645 (1987).

${ }^{10}$ K. C. Chiu, J. Ouazzani, and F. Rosenberger, "Mixed convection between horizontal plates-2. Fully developed flow,' Int. J. Heat Mass Transfer 30, 1655 (1987).

${ }^{11}$ J. M. Luijkx, J. K. Platten, and J. C. Legros, “'On the existence of ther- moconvective rolls, transverse to a superimposed mean Poiseuille flow," Int. J. Heat Mass Transfer 24, 803 (1981).

${ }^{12} \mathrm{~J}$. M. Luijkx, "Influence de la présence de parois latérales sur l'apparition de la convection libre, forcée et mixte,' Ph.D. thesis, University of MonsHainaut, Belgium, 1983.

${ }^{13}$ J. K. Platten and J. C. Legros, Convection in Liquids (Springer-Verlag, New York 1984), Chaps. 6 and 8.

${ }^{14}$ M. T. Ouazzani, "'Transferts thermiques et mécanique des écoulements de convection mixte," Ph.D. thesis, University of Mons-Hainaut, Belgium, 1991.

${ }^{15}$ S. S. Chen and A. S. Lavine, "Laminar, buoyancy induced flow structures in a bottom heated, aspect ratio 2 duct with throughflow," Int. J. Heat Mass Transfer 39, 1 (1996).

${ }^{16}$ M. T. Ouazzani, J. K. Platten, and A. Mojtabi, "Etude expérimentale de la convection mixte entre deux plans horizontaux à températures différentes-2," Int. J. Heat Mass Transfer 33, 1417 (1990).

${ }^{17}$ M. T. Ouazzani, J. K. Platten, and A. Mojtabi, “Intermittent patterns in mixed convection," Appl. Sci. Res. 51, 677 (1993).

${ }^{18}$ E. Schröder and K. Bühler, "Three-dimensional convection in rectangular domains with horizontal throughflow," Int. J. Heat Mass Transfer 38, 1249 (1995).

${ }^{19}$ H. R. Brand, R. J. Deissler, and G. Ahlers, "Simple model for the Bénard instability with horizontal flow near threshold," Phys. Rev. A 43, 4262 (1991).

${ }^{20}$ H. W. Müller, M. Tveitereid, and S. Trainoff, "Rayleigh-Bénard problem with imposed weak through-flow: Two coupled Ginzburg-Landau equations," Phys. Rev. E 48, 263 (1993).

${ }^{21}$ H. W. Müller, M. Lücke, and M. Kamps, "Convective patterns in horizontal flow,' Europhys. Lett. 10, 451 (1989).

${ }^{22}$ R. J. Deissler, "Spacially growing waves, intermittency and convective chaos in an open flow system," Physica D 25, 233 (1987).

${ }^{23}$ M. T. Ouazzani, J. K. Platten, H. W. Müller, and M. Lücke, "Etude de la convection mixte entre deux plans horizontaux à des températures différentes -3,' Int. J. Heat Mass Transfer 38, 875 (1995).

${ }^{24}$ H. W. Müller, "Thermische Konvection in horizontaler Scherströmung," Ph.D. thesis, University of Saarlandes in Sarrebruck, Germany, 1990.

${ }^{25}$ M. T. Ouazzani, J. P. Caltagirone, G. Meyer, and A. Mojtabi, "Etude numérique et expérimentale de la convection mixte entre deux plans horizontaux à températures différentes," Int. J. Heat Mass Transfer 32, 261 (1989).

${ }^{26}$ Below are given the numerical values corresponding to the experimental conditions of Refs. 14, 16, 17, 23 and used for comparisons in the present paper. Geometrical characteristics of the channel: length in the $x$ direction $L=115 \mathrm{~mm}$, width in the $y$ direction $l=15.05 \mathrm{~mm}$, height in the $z$ direction $h=4.15 \mathrm{~mm}$, longitudinal aspect ratio $A=L / h=27.7$, lateral aspect ratio $B=l / h=3.63$. Physical characteristics of water at $T=23^{\circ} \mathrm{C}$ : thermal diffusivity $\alpha=0.145 \times 10^{-6} \mathrm{~m}^{2} / \mathrm{s}$, thermal expansion coefficient $\beta=237.62 \times 10^{-6} \mathrm{~K}^{-1}$, kinematic viscosity $\nu=0.93 \times 10^{-6} \mathrm{~m}^{2} / \mathrm{s}$.

${ }^{27}$ M. Fortin and R. Glowinski, Méthodes de Lagrangien Augmenté, Applications á la Résolution Numérique de Problèmes ausc Limites, Collection Méthodes Mathématique de l'Informatique-9, Dunod editeur (Bordas, Paris, 1982).

${ }^{28}$ R. Glowinski, Numerical Methods for Non-linear Variational Problems, Springer Series in Computational Physics (Springer-Verlag, New York, 1984).

${ }^{29}$ K. J. Arrow, L. Hurwicz, and H. Uzawa, Studies in Nonlinear Programming (Stanford University Press, Stanford, CA, 1958).

${ }^{30}$ X. Nicolas, P. Traore, A. Mojtabi, and J. P. Caltagirone, "Augmented Lagrangian method and open boundary conditions in the 2D simulation of the Poiseuille-Bénard channel flow,' Int. J. Numerical Methods Fluids (in press).

${ }^{31}$ I. Orlanski, "A simple boundary condition for unbounded hyperbolic flows," J. Comput. Phys. 21, 251 (1976).

${ }^{32} \mathrm{P}$. Bergé, "Rayleigh-Bénard instability: Experimental findings obtained by light scattering and other optical methods,' in Fluctuation, Instabilities and Phase Transition, edited by T. Riste (NATO Advanced Study Institutes, Plenum Press, New York, 1975), Vol. B11, pp. 323-352.

${ }^{33}$ C. Normand, Y. Pomeau, and M. G. Velarde, "Convective instability: A physicists approach,” Rev. Mod. Phys. 49, 581 (1977).

${ }^{34}$ A. Schlüter, D. Lortz, and F. Busse, "On the stability of steady finite amplitude convection,” J. Fluid Mech. 23, 129 (1965). 